\title{
COORDINACIÓN INTERINSTITUCIONAL DE LOS SERVICIOS DE ATENCIÓN A LA PEQUEÑA INFANCIA EN LA RIOJA
}

\author{
Sylvia Sastre i Riba \\ Universidad de La Rioja \\ Felisa Bonachía Caballero \\ Consejería de Salud, Gobierno de La Rioja
}

\section{RESUMEN}

El objetivo del estudio es conocer el tipo y características de la coordinación entre los Centros de Atención Temprana en la Comunidad Autónoma de La Rioja.

Material y Método: Mediante un cuestionario mixto, construido "ad hoc", se ha obtenido la respuesta de $n=47$ profesionales de Centros de Atención a la Pequeña Infancia de La Rioja. Se han calculado, mediante el programa SPSS, las diferencias significativas $(p<0,05)$ entre las respuestas.

Los resultados muestran que: a) Los profesionales se coordinan por iniciativa propia en el tratamiento de casos; b) las medidas de Atención se coordinan entre profesionales del propio Centro y otros Centros (Servicios Sanitarios y Servicios Educativos) en menor medida; c) la dotación de recursos de atención temprana es irregular e insuficiente; d) se desconocen los objetivos y funciones de otros Centros/Servicios; e) hay falta de organización coordinada entre ellos.

Conclusiones: La estructura actual de los Servicios de atención temprana no es la adecuada e insuficiente para un servicio de calidad. Se requiere una coordinación institucionalizada reglamentada, con implicación de las instituciones intervinientes para su planificación.

PALABRAS CLAVE: Atención Temprana, Coordinación Interinstitucional, Pequeña Infancia.

\section{ABSTRACT}

The aim of this work is knowing the state of the art of the coordination measures among the Early Infancy Intervention Centers in the La Rioja's region .

Materials and method: data was extracted by a mixed questionnary constructed "ad hoc" and administered to $n=47$ professionals of Early 
Interventional Centres distributed according their geographical location: Rioja Alta, Rioja Centro, Rioja Baja. Significant differences among answers obtained was calculated using the SPSS program $(p<0.005)$ in relation to the conceptual axis designed.

Results show that: a) coordination among professionals is made by their own initiative in order to discuss individual treatments programmed; b) interventional procedures are best coordinated, significantly, among the professionals in the same interventional center; c) public resources distribution is differential, irregular and insufient relating to the geographical location; d) knowing of the working objectives and functions of other early interventional centers are unknown; e) there is a lack of organization among them,

Conclusion: the current structure of the Early Interventional Centers in La Rioja is poorly organized; oficial regulated coordination is necessary.

KEY WORDS: early intervention, infancy, interinstitutional coordination.

\section{INTRODUCCIÓN}

Los niños menores de seis años o población comprendida dentro de lo que se denomina "pequeña infancia" son los destinatarios de los Servicios de Atención Infantil. Las necesidades de salud de la pequeña infancia son de tipo biopsicosocial y constituyen la base de un desarrollo madurativo adecuado, según postulados de la Organización Mundial de la Salud (O.M.S., 1999). Así pues, todos los elementos del medio físico y social actúan sobre los aspectos biológicos y psíquicos de la vida, en los diversos grados de su crecimiento y desarrollo, que no son estáticos, sino que son "vivos" y cambiables por la experiencia (Villuendas, 1989). Por ello, el entorno humano directo juega un rol esencial en el conocimiento y la satisfacción de estas necesidades, siendo responsable de garantizar los recursos y los servicios idóneos para ello, ya que estos primeros años son un momento único, crucial para el desarrollo con unas características y unas posibilidades que no tienen comparación con ninguna otra etapa de la vida (Gutiez, 2005). Pero el desarrollo humano puede verse interferido por factores de riesgo psicobiológico desde la etapa prenatal, por eso es importante detectarlos tan pronto como sea posible, para establecer una intervención temprana que palie sus consecuencias y optimice la capacidad de desarrollo y de bienestar, posibilitando, de la forma más completa, su autonomía personal y su integración en el medio familiar, escolar y social (Millá, 2003).

Esta intervención temprana se lleva a cabo en los Centros de Desarrollo Infantil y Atención Temprana (CDIAT) por equipos interdisciplinares que abordan de forma individualizada y global la atención específica que se requiere en cada caso, de acuerdo a las pautas del "calendario de desarrollo". Es en estos centros donde se hace imprescindible la coordinación del entorno del niño y la familia con los distintos servicios que participan en la atención (Pegenaute, 2003).

Los equipos interdisciplinares (pediatras, psicólogos, psicopedagogos, logopedas, fisiatras, maestros de educación infantil, médicos de salud mental, etc) deben actuar de forma coordinada en la atención al desarrollo integral del niño, teniendo en cuenta a la familia y el entorno (GAT, 2000).

Las instituciones que intervienen en este proceso de Atención a la Pequeña Infancia son: Sociales o de Trabajo Social, actuando principalmente en situaciones 
carenciales o de marginación (Anguera, 1995), Sanitarias, Educativas y también las Asociaciones, procediendo de forma coordinada para planificar, gestionar y evaluar la Atención Temprana en su ámbito de competencia.

En un modelo integral de intervención se deben abordar completamente los distintos niveles de actuación en prevención primaria (estudios para la detección riesgos y/o patologías en la población infantil), secundaria (fundamentalmente detección, valoración y diagnóstico) y terciaria (intervención propiamente dicha) (García, 2002).

La diversidad de profesionales y disciplinas favorece la calidad de la intervención, pero añade el problema de la coordinación de profesionales y equipos (Soriano, 1999), incluso entre las instituciones implicadas, pero coordinarse no es sólo hablar de forma ordenada, sino que implica un plan de acción conjunta (Ullman y Lázaro, 2007).

En España, cada Comunidad Autónoma aborda de diferente manera la Atención Temprana y establece una configuración propia de la estructura de los CDIAT.

En la Comunidad Autónoma de La Rioja, los Servicios de Atención a la Pequeña Infancia, que prestan atención a los niños de 0-6 años de edad, están comprendidos en la red asistencial pública dependiente de las Consejerías de Salud, Educación y Juventud, Familia y Servicios Sociales. También se cuenta con Asociaciones orientadas a la Atención. Estos Centros/Servicios se distribuyen en La Rioja de la siguiente manera:

Servicios Sanitarios:

- Servicios de Pediatría hospitalarios

- Servicios de Pediatría de Atención Primaria

- Servicio de Salud Mental Infanto-juvenil

- Servicio de Prevención y Promoción de la Consejería de Salud

- Gerencia de Atención Primaria (Servicio Riojano de Salud)

Servicios Sociales:

- Servicio de Mujer, Familia e Infancia: Área de Protección de Menores

- Servicio de Acción Social: Centro Base de Minusválidos

- Dirección General de Recursos de Servicios Sociales: Guarderías ${ }^{[*} /$ Escuelas $^{2}$ Infantiles

Servicios Educativos:

- Colegios públicos: Centros Escolares Infantiles

- Equipo de Atención Temprana

- Equipos de Orientación Educativa y Psicopedagógica (EOEP)

Asociaciones: (se detallan las que disponen de servicios de atención a niños de 0-6 años).

- Aspace-Rioja (Asociación de atención a las Personas con parálisis cerebral de La Rioja)

- ARPA (Asociación Riojana para el Autismo)

- ARSIDO (Asociación Riojana para el Síndrome de Down)

[*] Se señala la inadecuación del término guarderías que no se corresponde con las tendencias actuales en Atención Temprana. 
- ONCE (Organización nacional de Ciegos)

También existen asociaciones de padres de niños con limitaciones y/o alteraciones en el desarrollo que no ofrecen servicios de atención.

En suma, en La Rioja existen 144 Centros institucionales de Atención a la Pequeña Infancia para una población entre 0-6 años de 18.991 (Registro de Tarjeta Sanitaria Individual, julio 2005). Estos Centros se hallan distribuidos en tres áreas geográficas: Rioja Alta, Rioja Centro y Rioja Alta y, a su vez, en un total de 18 zonas básicas de salud.

El objetivo del trabajo consiste en conocer el tipo y características de la coordinación que existe entre los mencionados Centros de Atención a la Pequeña Infancia de La Rioja.

\section{MÉTODO}

\section{1.- Participantes}

Profesionales, responsables de la Atención a la Pequeña Infancia en la Rioja, titulados universitarios (licenciados, o diplomados) que trabajan en los diferentes Centros/Servicios seleccionados y que pertenecen a Instituciones Sanitarias, Sociales, Educativas y, también, a las Asociaciones; $\mathrm{n}=47$.

\section{2.- Instrumento}

Para la extracción de datos, se ha elaborado "ad hoc" un cuestionario mixto de preguntas abiertas y cerradas, con un total de $\mathrm{n}=29$ preguntas, estructurado en varios ejes:

a) Características descriptivas de la muestra

b) Definición y necesidades de la Atención Temprana

c) Programas de Atención a la Pequeña Infancia en La Rioja

d) Coordinación entre los profesionales y los niveles de Atención Temprana

e) Recursos institucionales para la Atención a la Pequeña Infancia

f) Estructura de los Servicios de Atención a la Pequeña Infancia en La Rioja

g) Formación de los profesionales en su ámbito de actuación

\section{3.- Procedimiento}

Los pasos sistemáticos seguidos en el estudio han sido:

a) Selección y extracción de la muestra bajo los siguientes criterios:

- Los centros seleccionados se clasifican según las tres áreas de La Rioja (Alta, Centro y Baja), en las cuales, a su vez, que se incluyen las zonas básicas de salud correspondientes.

- Que existan, al menos, Servicios Sanitarios y Servicios Educativos en las zonas básicas

- En los Servicios sanitarios debe haber atención pediátrica, al menos, el tiempo correspondiente a media jornada de trabajo.

- En las zonas en las que existen varios centros docentes, se selecciona un centro público y otro concertado

- En la ciudad de Logroño se seleccionan tres zonas básicas de las seis que la componen en el momento del estudio, con las características sociodemográficas más representativas. 
b) Elaboración "ad hoc" y valoración interjueces previa, de un cuestionario mixto con preguntas abiertas y cerradas.

c) Redacción y envío de una carta de presentación e invitación a la participación en el estudio.

d) Entrega del cuestionario en los centros seleccionados $(n=47)$ y recogida tras el plazo determinado.

e) Análisis estadístico inferencial basado en la comparación de proporciones y frecuencias mediante la prueba chi cuadrado, con el fin de conocer la existencia, o no, de diferencias significativas entre los tipos de respuestas recogidas. Para ello se ha utilizado el programa Estadístico SPSS para Windows, versión 10.0.

f) Tabulación de los datos obtenidos en las preguntas abiertas del cuestionario, se categorizan las respuestas según la afinidad de las valoraciones expresadas, obteniendo bloques de contenido a los que se asigna un valor numérico con objeto de cuantificar las categorías de respuestas resultantes.

\section{RESULTADOS}

\section{1.- Características descriptivas de la muestra}

La participación de los profesionales implicados en la Atención a la Pequeña Infancia es mayoritariamente de género femenino. La experiencia profesional es de más de 15 años. La titulación académica más frecuente es la de Educador - Maestro de Educación Infantil, seguido de Médico - Pediatra - Rehabilitador - Psiquiatra.

\section{2.- Definición y necesidades de Atención Temprana}

Los profesionales definen, significativamente, la Atención Temprana como las alteraciones y/o riesgo en el desarrollo de los niños e intervención en dicho desarroIlo, en contraposición con la idea de discapacidad. Para su análisis, en la Tabla $\mathrm{n}^{\circ} 1$ se representan las categorizaciones de las respuestas con rasgos similares, describiendo los siguientes bloques de contenido:

Bloque 1 - Detección-Prevención

Bloque 2 - Discapacidad

Bloque 3 - Alteraciones/riesgo en el desarrollo

Bloque 4 - Atención-Intervención

Bloque 5 - Seguimiento del desarrollo en todos los niños

Bloque 6 - No sabe

Bloque 7 - Otras respuestas

Desde una perspectiva exploratoria, se realiza un análisis de contenido diferenciando las categorías encontradas en la definición estandarizada del concepto de Atención Temprana, según el Libro Blanco (GAT, 2000) (Ver Tablas $n^{\circ} 2$ y $n^{\circ} 3$ ), e incluyendo en ellas las mencionadas por los participantes. Estas categorías son:

- Seguimiento del desarrollo infantil en todos los niños

- Riesgo en el desarrollo infantil

- Discapacidad

- Atención-Intervención

- Prevención-Detección 
- Entorno-Familia

- Atención global

- Multidisciplinariedad

- Edad de actuación de 0 - 6 años

En las Tablas 1 y 2 se recogen los resultados observados en esta prospección conceptual, referidas a las categorías descritas.

Tabla $n^{\circ}$ 1. Definición de Atención Temprana

\begin{tabular}{lcccc}
\hline Bloques de Contenido & Frecuencia & Porcentaje & $\%$ Válido & $\mathbf{X}^{2}$ \\
\hline $3-4$ & 13 & 27,7 & 28,3 &, 000 \\
\hline $4-5$ & 8 & 17 & 17,4 & \\
\hline $1-3-4$ & 8 & 17 & 17,4 & \\
\hline $1-3$ & 4 & 8,5 & 8,7 & \\
\hline $2-4$ & 2 & 4,3 & 4,3 \\
\hline 5 & 2 & 4,3 & 4,3 \\
\hline $1-4-5$ & 2 & 4,3 & 4,3 \\
\hline $1-5$ & 2 & 4,3 & 4,3 \\
\hline $2-3-4$ & 1 & 2,1 & 2,2 \\
\hline 7 & 1 & 2,1 & 2,2 \\
\hline 6 & 1 & 2,1 & 2,2 \\
\hline $1-2$ & 1 & 2,1 & 2,2 \\
\hline $3-4-5$ & 1 & 2,1 & 2,2 \\
\hline Total & 46 & 97,9 & 100 \\
\hline V. Perdidos & 1 & 2,1 & \\
\hline $\mathrm{N}$ & 47 & 100 & \\
\hline
\end{tabular}

Tabla $\mathbf{n}^{\circ}$ 2. Categorías que definen la Atención Temprana

Categorías que definen la Atención Temprana

Atención-Intervención en las alteraciones del desarrollo infantil

Riesgo de alteraciones en el desarrollo infantil

Prevención-Detección de las alteraciones/riesgo del desarrollo infantil

Seguimiento del desarrollo infantil en todos los niños

Seguimiento del desarrollo infantil de 0-6 años

Atención global en la infancia

Multidisciplinariedad en el seguimiento del desarrollo infantil

Discapacidad en la infancia

Participación de la familia y el entorno en el desarrollo infantil 3
Frecuencia Porcentaje \% Válido

$35 \quad 74,5 \quad 76,1 \%$

\begin{tabular}{ccc}
27 & 57,4 & $58,7 \%$ \\
\hline 17 & 36,2 & $37 \%$
\end{tabular}

15

11

31,9

$32,6 \%$

$23,4 \quad 23,9 \%$

7

4

14,9

$15,2 \%$

8,5

$8,7 \%$

4

8,5

$8,7 \%$

3

6,4

$6,5 \%$ 
Tabla $\mathbf{n}^{\circ}$ 3. Categorías de la definición de Atención Temprana. Libro Blanco (GAP, 2000)

\begin{tabular}{|c|c|c|c|c|c|}
\hline Pregunta 1 & Frecuencia & & Porcentaje & \% Válido & $X^{2}$ \\
\hline Seguimiento del & No & 31 & 66 & 67,4 & 018 \\
\hline desarrollo infantil & Sí & 15 & 31,9 & 32,6 & \\
\hline \multirow{3}{*}{ en todos los niños } & Total & 46 & 97,9 & 100 & \\
\hline & Valores perdidos & 1 & 2,1 & & \\
\hline & $\mathrm{N}$ & 47 & 100 & 100 & \\
\hline Pregunta 1 & Frecuencia & & Porcentaje & \% Válido & $X^{2}$ \\
\hline Riesgo en el & Sí & 27 & 57,4 & 58,7 & 238 \\
\hline \multirow[t]{4}{*}{ desarrollo infantil } & No & 19 & 40,4 & 41,3 & \\
\hline & Total & 46 & 97,9 & 100 & \\
\hline & Valores perdidos & 1 & 2,1 & & \\
\hline & $\mathrm{N}$ & 47 & 100 & 100 & \\
\hline Pregunta & Frecuencia & & Porcentaje & \% Válido & $\mathbf{X}^{2}$ \\
\hline \multirow[t]{5}{*}{ Discapacidad } & No & 42 & 89,4 & 91,3 & ,000 \\
\hline & Sí & 4 & 8,5 & 8,7 & \\
\hline & Total & 46 & 97,9 & 100 & \\
\hline & Valores perdidos & 1 & 2,1 & & \\
\hline & $\mathrm{N}$ & 47 & 100 & 100 & \\
\hline Pregunta 1 & Frecuencia & & Porcentaje & \% Válido & $\mathbf{X}^{2}$ \\
\hline \multirow{5}{*}{ Atención - Intervención } & Sí & 35 & 74,5 & 76,1 & ,000 \\
\hline & No & 11 & 23,4 & 23,9 & \\
\hline & Total & 46 & 97,9 & 100 & \\
\hline & Valores perdidos & 1 & 2,1 & & \\
\hline & $\mathrm{N}$ & 47 & 100 & 100 & \\
\hline Pregunta 1 & Frecuencia & & Porcentaje & \% Válido & $X^{2}$ \\
\hline \multirow[t]{5}{*}{ Prevención - Detección } & No & 29 & 61,7 & 63 & ,077 \\
\hline & Sí & 17 & 36,2 & 37 & \\
\hline & Total & 46 & 97,9 & 100 & \\
\hline & Valores perdidos & 1 & 2,1 & & \\
\hline & $\mathrm{N}$ & 47 & 100 & 100 & \\
\hline Pregunta 1 & Frecuencia & & Porcentaje & \% Válido & $\mathrm{X}^{2}$ \\
\hline \multirow[t]{5}{*}{ Entorno - Familia } & No & 43 & 91,5 & 93,5 &, 000 \\
\hline & Sí & 3 & 6,4 & 6,5 & \\
\hline & Total & 46 & 97,9 & 100 & \\
\hline & Valores perdidos & 1 & 2,1 & & \\
\hline & $\mathrm{N}$ & 47 & 100 & 100 & \\
\hline
\end{tabular}




\begin{tabular}{llrccc}
\hline Pregunta 1 & Frecuencia & & Porcentaje & \% Válido & $\mathbf{X}^{2}$ \\
\hline Atención Global & No & 39 & 83 & 84,8 &, 000 \\
& Sí & 7 & 14,9 & 15,2 & \\
& Total & 46 & 97,9 & 100 & \\
& Valores perdidos & 1 & 2,1 & & \\
& N & 47 & 100 & 100 & \\
\hline Pregunta 1 & Frecuencia & & Porcentaje & \% Válido & $\mathbf{X}^{2}$ \\
\hline Multidisciplinariedad & No & 42 & 89,4 & 91,3 &, 000 \\
& Sí & 4 & 8,5 & 8,7 & \\
& Total & 46 & 97,9 & 100 & \\
& Valores perdidos & 1 & 2,1 & & \\
& N & 47 & 100 & 100 &, 000 \\
\hline Pregunta 1 & Frecuencia & & & & \\
\hline Edad: 0-6años & No & 35 & 74,5 & 76,1 & \\
& Sí & 11 & 23,4 & 23,9 & \\
& Total & 46 & 97,9 & 100 & \\
& Valores perdidos & 1 & 2,1 & & \\
& N & 47 & 100 & 100 & \\
& & & & &
\end{tabular}

\section{3.- Programas de Atención a la Pequeña Infancia}

Los profesionales solicitan a la Administración, para una correcta atención, una buena organización de la Atención Temprana, con mayor disponibilidad de recursos y mayor coordinación entre ellos. Para el análisis de esta cuestión, se categorizan las respuestas en los siguientes bloques de contenido:

Bloque 1 - Más recursos

Bloque 2 - Mayor coordinación

Bloque 3 - Fomento de la formación-investigación

Bloque 4 - Organización de la Atención Temprana

Bloque 5 - Otras respuestas

Los resultados se representan en la Tabla $\mathrm{n}^{\circ} 4$. 
Tabla $\mathbf{n}^{\circ}$ 4. ¿Qué solicitaría a la Administración para una correcta atención?

\begin{tabular}{lcccc}
\hline Bloques de Contenido & Frecuencia & Porcentaje & \% Válido & $\mathbf{X}^{2}$ \\
\hline 4 & 7 & 14,9 & 21,2 &, 040 \\
\hline 1 & 7 & 14,9 & 21,2 & \\
\hline 2 & 3 & 6,4 & 9,1 & \\
\hline $1-2-4$ & 3 & 6,4 & 9,1 & \\
\hline $1-2$ & 3 & 6,4 & 9,1 \\
\hline $1-4$ & 2 & 4,3 & 6,1 \\
\hline $1-2-3-4$ & 2 & 4,3 & 6,1 \\
\hline $1-3$ & 1 & 2,1 & 3 \\
\hline $1-3-4$ & 1 & 2,1 & 3 \\
\hline 5 & 1 & 2,1 & 3 \\
\hline $2-3-4$ & 1 & 2,1 & 3 \\
\hline $2-3$ & 1 & 2,1 & 3 \\
\hline $1-2-3$ & 1 & 2,1 & 3 \\
\hline Total & 33 & 70,2 & 100 \\
\hline Valores perdidos & 14 & 29,8 & \\
\hline N & 47 & 100 & \\
\hline
\end{tabular}

De acuerdo con los resultados obtenidos (ver Tabla $n^{\circ}$ 5), en La Rioja, los Planes y Programas institucionales se conocen poco. En el centro de trabajo, los profesionales realizan programas individualizados de promoción y prevención y también educacionales. Se utilizan escalas de desarrollo diferentes en cada institución, lo que muestra que hay diferencias en los instrumentos de medida o evaluación, así como falta de homogeneidad (Tabla $\left.n^{\circ} 6\right)$.

Tabla $n^{\circ}$ 5. Programas y Planes de Atención a la Pequeña Infancia en La Rioja

\begin{tabular}{llcccc}
\hline Pregunta 3 & Frecuencia & & Porcentaje & \% Válido & $\mathbf{X}^{\mathbf{2}}$ \\
\hline Conoce el Libro Blanco & No & 22 & 46,8 & 56,4 &, 423 \\
de Atención Temprana & Sí & 17 & 36,2 & 43,6 & \\
& total & 39 & 83 & 100 & \\
& Valores perdidos & 8 & 17 & & \\
& N & 47 & 100 & 100 & \\
\hline Conoce el Programa de & Sí & 21 & 44,7 & 52,5 &, 752 \\
Atención al Niño Sano & No & 19 & 40,4 & 47,5 & \\
& total & 40 & 85,1 & 100 & \\
& Valores perdidos & 7 & 14,9 & & \\
& N & 47 & 100 & 100 & \\
\hline Conoce el Plan Integral & No & 21 & 44,7 & 56,8 & \\
de Infancia & Sí & 16 & 34 & 43,2 & \\
& total & 37 & 78,7 & 100 & \\
& Valores perdidos & 10 & 21,3 & & \\
& N & 47 & 100 & 100 & \\
\hline
\end{tabular}




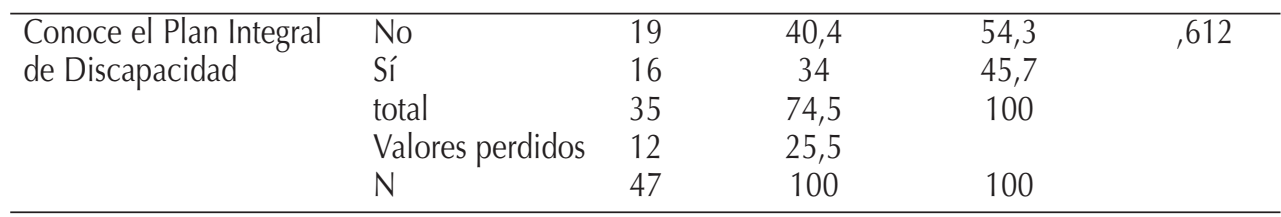

Tabla $n^{\circ}$ 6. "Escalas de Desarrollo" utilizadas según la Institución

\begin{tabular}{|c|c|c|c|c|c|}
\hline $\begin{array}{l}\text { "Escalas de } \\
\text { Desarrollo" }\end{array}$ & Asociaciones & $\begin{array}{l}\text { Servicios } \\
\text { Sociales }\end{array}$ & $\begin{array}{c}\text { Servicios } \\
\text { Educativos }\end{array}$ & $\begin{array}{l}\text { Servicios } \\
\text { Sanitarios }\end{array}$ & $\begin{array}{c}\text { Frecuencia } \\
\text { total }\end{array}$ \\
\hline WPSSI-R & & & 2 & & 2 \\
\hline WISC-R & 1 & & & & 1 \\
\hline BADyG & 1 & & & & 1 \\
\hline$\underline{\mathrm{EPI}}$ & 1 & & & & 1 \\
\hline $\begin{array}{l}\text { PAC: } \\
\text { M/P.A.C.-1; P-E-I }\end{array}$ & 1 & & & & 1 \\
\hline Rueda del Desarrollo & 1 & & & & 1 \\
\hline $\begin{array}{l}\text { Escalas de Observación } \\
\text { Sistemática }\end{array}$ & 1 & & & & 1 \\
\hline Brunet-Lezine & 1 & 1 & 1 & 2 & 5 \\
\hline Batelle & 3 & 1 & 3 & & 7 \\
\hline Leonhardt & 1 & & & & 1 \\
\hline Bayley & 1 & 1 & & & 2 \\
\hline Portage & 2 & 1 & 4 & & 7 \\
\hline Columbia & 1 & & & & 1 \\
\hline Mc Carthy & 1 & & 1 & & 2 \\
\hline $\mathrm{EOD}$ & & & 1 & 1 & \\
\hline $\begin{array}{l}\text { Observación de dibujos: } \\
\text { Test de la casa-árbol- } \\
\text { figura humana }\end{array}$ & & & 1 & & 1 \\
\hline Bender & & & 1 & & 1 \\
\hline Orbegozo & & & & 2 & 2 \\
\hline Haizea Llevant & & & & 8 & 8 \\
\hline Denver & & & & $\$$ & \\
\hline Currículo Carolina & & & & 1 & 1 \\
\hline Leiter-R & & & & 11 & \\
\hline Gesell & & & & 11 & \\
\hline
\end{tabular}




\section{4.- Coordinación entre profesionales y los niveles de Atención Temprana}

Los profesionales declaran que se coordinan por iniciativa propia en el tratamiento de los casos. La coordinación se realiza principalmente entre los profesionales del propio centro y, también, con otros centros, de acuerdo con los resultados recogidos en la Tabla $n^{\circ} 7$.

Tabla $\mathbf{n}^{\circ}$ 7. Niveles de coordinación durante el seguimiento del proceso de atención

\begin{tabular}{lcccc}
\hline Bloques de Contenido & Frecuencia & Porcentaje & $\%$ Válido & $\mathbf{X}^{\mathbf{2}}$ \\
\hline $1-2$ & 21 & 44,7 & 50 &, 000 \\
\hline $1-2-3$ & 14 & 29,8 & 33,3 & \\
\hline 1 & 5 & 10,6 & 11,9 & \\
\hline 2 & 1 & 2,1 & 2,4 & \\
\hline $1-2-3-4$ & 1 & 2,1 & 2,4 & \\
\hline Total & 42 & 89,4 & 100 & \\
\hline Valores perdidos & 5 & 10,6 & & \\
\hline $\mathrm{N}$ & 47 & 100 & & \\
\hline
\end{tabular}

\section{5.- Recursos institucionales para la atención a la pequeña Infancia}

Los recursos actuales son significativamente insuficientes para el seguimiento de los casos y su evaluación (ver Tabla $n^{\circ}$ 8). Por otra parte, los recursos están dotados de forma irregular y también es, en algunos casos, insuficiente, según la distribución de la población de 0- 6 años de edad.

Tabla $\mathbf{n}^{\circ}$ 8. ¿Son suficientes los recursos actuales para el seguimiento y evaluación de los casos?

\begin{tabular}{|c|c|c|c|c|c|}
\hline Pregunta 16 & \multicolumn{2}{|l|}{ Frecuencia } & \multirow{2}{*}{$\begin{array}{c}\text { Porcentaje } \\
78,7\end{array}$} & \multicolumn{2}{|c|}{$\%$ Válido $X^{2}$} \\
\hline \multirow{5}{*}{$\begin{array}{l}\text { Considera suficientes } \\
\text { los recursos actuales } \\
\text { para un seguimiento } \\
\text { de los casos y su } \\
\text { evaluación }\end{array}$} & No & 37 & & 90,2 & ,000 \\
\hline & Sí & 4 & 8,5 & 9,8 & \\
\hline & Total & 41 & 87,2 & 100 & \\
\hline & Valores perdidos & 6 & 12,8 & & \\
\hline & $\mathrm{n}$ & 74 & 100 & & \\
\hline
\end{tabular}

\section{6.- Estructura de los Servicios de Atención a la Pequeña Infancia}

De acuerdo con las respuestas obtenidas, los profesionales de Atención Temprana no conocen los objetivos y funciones de otros Centros/Servicios ni disponen de información suficiente sobre el funcionamiento de los servicios y su estructura según se recoge en la Tabla $n^{\circ}$ 9. Cuando los profesionales precisan realizar interconsultas de Atención Temprana, para la atención de los casos, éstas se realizan significativamente con el Servicio de Neuropediatría del Hospital San Millán, tal como se recoge en las Tablas $\mathrm{n}^{\circ} 10$ y 11. 
Tabla $\mathbf{n}^{\circ}$ 9. ¿Se dispone de información suficiente sobre el funcionamiento de los servicios y su estructura?

\begin{tabular}{|c|c|c|c|c|c|}
\hline Pregunta 13 & Frecuencia & & Porcentaje & \% Válido & $\mathbf{X}^{2}$ \\
\hline \multirow{5}{*}{$\begin{array}{l}\text { ¿Se dispone de } \\
\text { información suficiente } \\
\text { sobre el funcionamiento } \\
\text { de los servicios y } \\
\text { su estructura? }\end{array}$} & No & 44 & 96,6 & 93,6 & ,000 \\
\hline & Sí & 3 & 6,4 & 6,4 & \\
\hline & Total & 47 & 100 & 100 & \\
\hline & Valores per & & & & \\
\hline & $\mathrm{n}$ & 47 & 100 & 100 & \\
\hline
\end{tabular}

Tabla $\mathbf{n}^{0}$ 10. ¿ Utiliza interconsultas de Atención Temprana?

\begin{tabular}{llrccc}
\hline Pregunta 7 & Frecuencia & & Porcentaje & \% Válido & $\mathbf{X}^{\mathbf{2}}$ \\
\hline \multirow{2}{*}{$\begin{array}{l}\text { Utiliza } \\
\text { Interconsultas }\end{array}$} & Sí & 34 & 72,3 & 73,9 &, 001 \\
\cline { 2 - 7 } de Atención & No & 12 & 25,5 & 26,1 & \\
\cline { 2 - 7 } Temprana? & Total & 46 & 97,9 & 100 & \\
\cline { 2 - 6 } & Valores perdidos & 1 & 2,1 & & 100 \\
\cline { 2 - 6 } & Total & 47 & 100 & 100 \\
\hline
\end{tabular}

Bloques de contenido definidos para la Tabla $n^{\circ} 10$

Servicio de Pediatría del Hospital San Millán (Neuropediatría)

Servicio de Pediatría de la Fundación Hospital de Calahorra

Servicios de Pediatría de Atención Primaria (Centros de Salud)

Unidad de Salud Mental infanto-juvenil

Equipo de Atención Temprana

FEIDS (Federación Española de Instituciones para el Síndrome de Down)-ARSIDO

Equipos de Orientación Educativa y Psicopedagógica

Centros Escolares Infantiles

Centro Base de Minusválidos

Centros privados y/o concertados

Departamento de Menores de la Consejería de Servicios Sociales

No tienen acceso

\section{7.- Formación de los profesionales}

Los participantes consideran necesaria una formación específica para intervenir en Atención Temprana, formación que los profesionales declaran realizar fuera del horario de trabajo. En cuanto a la productividad y difusión del trabajo realizado, la mayoría de profesionales no ha realizado publicaciones en los últimos tres años, caso afirmativo, son de ámbito autonómico y nacional. 
Tabla $n^{\circ}$ 11. Centros adonde se dirigen cuando se necesitan interconsultas de Atención Temprana

\begin{tabular}{lcccc}
\hline Bloques de Contenido & Frecuencia & Porcentaje & $\%$ Válido & $\mathbf{X}^{\mathbf{2}}$ \\
\hline 1 & 10 & 21,3 & 26,3 &, 000 \\
\hline 5 & 6 & 12,8 & 15,8 & \\
\hline 7 & 4 & 8,5 & 10,5 & \\
\hline $1-5-10$ & 2 & 4,3 & 5,3 \\
\hline $1-2$ & 2 & 4,3 & 5,3 \\
\hline $1-4$ & 2 & 4,3 & 5,3 \\
\hline $3-5-7$ & 2 & 4,3 & 5,3 \\
\hline $1-9$ & 1 & 2,1 & 2,6 \\
\hline 6 & 1 & 2,1 & 2,6 \\
\hline $1-4-5$ & 1 & 2,1 & 2,6 \\
\hline 3 & 1 & 2,1 & 2,6 \\
\hline $3-5$ & 1 & 2,1 & 2,6 \\
\hline $1-2-5-8-9$ & 1 & 2,1 & 2,6 \\
\hline $1-7-9$ & 1 & 2,1 & 2,6 \\
\hline 12 & 1 & 2,1 & 2,6 \\
\hline $1-3-5-9-10-11$ & 1 & 2,1 & 2,6 \\
\hline $1-6$ & 1 & 2,1 & 2,6 \\
\hline Total & 38 & 80,9 & 100 \\
\hline Valores perdidos & 9 & 19,1 & \\
\hline $\mathrm{N}$ & 47 & 100 & \\
\hline
\end{tabular}

\section{CONCLUSIONES}

El rasgo más característico de los profesionales responsables de la Atención a la Pequeña Infancia en La Rioja es que son mayoritariamente de género femenino y que aportan una gran experiencia y cualificación, lo que indica una actitud vocacional de las mujeres en la participación de la atención directa a los niños.

Los profesionales definen la Atención Temprana como las alteraciones y/o riesgo en el desarrollo de los niños así como la atención/intervención en dicho desarrollo. Estos conceptos van en contraposición con la idea de discapacidad, lo que nos sitúa en una visión muy actualizada, que incluso supera los postulados del Libro Blanco (GAT, 2000), en cuanto que se estima necesaria una tarea constante en la vigilancia y detección de las posibles limitaciones y/o riesgo de tenerlas mediante controles establecidos a modo de calendario de desarrollo. Para conseguirlo, solicitarían de la Administración una buena organización de la Atención Temprana con mayor disponibilidad de recursos y mayor coordinación entre ellos.

La atención se realiza en los centros mediante programas individualizados de promoción-prevención y educacionales consensuados entre el equipo multidisciplinar y 
también con otros centros, ya que los programas institucionales se conocen poco. Entre las instituciones de Atención a la Pequeña Infancia en La Rioja, existe heterogeneidad en cuanto a las "Escalas de Desarrollo" que se utilizan, lo que indica la necesidad de protocolizar este aspecto, pues su inclusión homogeneizada en la práctica habitual de la Atención servirá para una detección de riesgos y/o alteraciones más fiable.

Los profesionales realizan, por iniciativa propia, una "puesta en común" de los casos tratados, aunque el intercambio de información entre diferentes departamentos es puntual. Significa que existe una falta de coordinación multidisciplinar e institucional como causa principal de la mencionada falta de homogeneización en la forma de actuar.

La configuración actual de la estructura de los servicios de Atención Temprana en La Rioja no es la adecuada y es insuficiente para prestar un servicio de calidad tanto en el seguimiento de los casos como en su evaluación, dando lugar a que haya casos sin resolver, principalmente en niños con dificultades leves.

Los servicios sanitarios son de referencia obligada en la coordinación de la Atención a la Pequeña Infancia, lo que sugiere un liderazgo implícito sobre las otras instituciones intervinientes en este tipo de atención.

En suma, se requiere una coordinación institucionalizada, reglamentada y con implicación de las instituciones intervinientes para la planificación de la Atención Temprana en La Rioja y su incidencia en la optimización de la calidad del desarrollo de la pequeña infancia atendida en ellos.

\section{BIBLIOGRAFÍA}

Anguera, M.T., Blanco, A., Losada, J.L., Montilla, Ma D., De Armas M. (1995). Los profesionales del Sistema de Atención Social a la Infancia. Realidad y demandas. Madrid. Ministerio de Asuntos Sociales.

García, F.A. (2002). Atención Temprana: elementos para el desarrollo de un Modelo Integral de Intervención. Bordón, 54, (1), 35-52.

Grupo de Atención Temprana (GAT) (2000). Libro Blanco de la Atención Temprana. Madrid: Ministerio de Trabajo y Servicios Sociales. Real Patronato de Prevención y de Atención a Personas con Minusvalía.

Gutiez, P. (2005). Atención Temprana. En P. Gutiez, (ed), Atención Temprana. Prevención, detección e intervención en el desarrollo (0-6 años) y sus alteraciones, (pp. 3-61): Madrid: Editorial Complutense.

Millá, Ma G. (2003). Atención Temprana: Definición y objetivos. Minusval. № especial, marzo, 48-51.

O.M.S. (1999). Salud 21. El marco político de salud para todos de la Región Europea de la O.M.S. Madrid: Ministerio de Sanidad y Consumo.

Pegenaute, F. (2003). Centros de Desarrollo Infantil y Atención Temprana. Minusval. $\mathrm{N}^{\circ}$ especial, marzo, 63-66.

Soriano, V. (1999). Intervención Temprana en europa: Organización de Servicios y Asistencia a los Niños y sus Familias. Tendencias en 17 Países Europeos. Agencia europea para el Desarrollo de la Educación Especial. (Trad. española Javier Velarte). Madrid. Real Patronato de Prevención y de Atención a Personas con minusvalía. 
Ullman, S., Lázaro, E. (2007). Coordinación entre centros escolares, atención temprana y familias. En M. Vidal (Coordinadora). Estimulación Temprana (De 0 a 6 años). Desarrollo de capacidades, valoración y programas de intervención. Primera parte: Perspectiva histórico-científico-social de la estimulación temprana, (pp 105-131). Madrid. CEPE.

Villuendas, M.D., (1989). Vida Psíquica y Atención Temprana. Fundamentos Psicopedagógicos, Metodológicos; Intervenciones y Recursos. Actas del Congreso Internacional de Educación Infantil. Madrid. 